Progress in Flight Physics 5 (2013) 363-376

DOI: $10.1051 /$ eucass/201305363

(C) Owned by the authors, published by EDP Sciences, 2013

\title{
EXPERIMENTAL AND NUMERICAL STUDY OF DUAL BELL NOZZLE FLOW
}

\author{
C. Génin ${ }^{1}$, R. Stark ${ }^{1}$, O. Haidn ${ }^{1}$, K. Quering ${ }^{2}$, \\ and M. Frey ${ }^{2}$ \\ ${ }^{1}$ German Aerospace Center - DLR, Institute for Space Propulsion \\ Hardthausen 74139, Germany \\ ${ }^{2}$ Astrium Space Transportation \\ Munich 81663, Germany
}

\begin{abstract}
The dual bell is a nozzle concept for altitude adaption. The flow separates at the contour inflection in sea level mode in a mainly controlled and symmetrical way, reducing the side load generation and increasing the thrust. The transition to altitude mode is reached when the flow suddenly attaches to the extension for an improved altitude thrust. The conditions of this transition and its evolution are the key for the study of dual bell nozzles. For a better understanding of the flow behavior, a two-dimensional (2D) subscale dual bell model has been designed and tested at the German Aerospace Center (DLR). The tests were divided into two campaigns and performed under cold and hot flow conditions. The evolution of the shock system at the inflection during the transition was observed using schlieren optics. The planar nozzle was tested under various conditions in pressure and temperature. Both test campaigns have been recalculated in cooperation with Astrium. Numerical and experimental results are presented.
\end{abstract}

\section{INTRODUCTION}

The main stage engine of the current launchers using parallel configuration (e.g., the European launcher Ariane 5) has to be ignited at ground for security reason. This imposes the limitation of the nozzle area ratio, reducing the potential overall performance. The nozzle works overexpanded at sea level and strongly underexpanded at altitude. Various concepts of altitude adaptive nozzles have been proposed in the literature to circumvent this limitation. The dual bell nozzle offers a very promising alternative to conventional nozzles [1].

A contour inflection links the base nozzle and the extension (Fig. 1) and forces the flow to a controlled and symmetrical separation in sea level mode. The side load generation is limited and the thrust increased. At a certain altitude, 

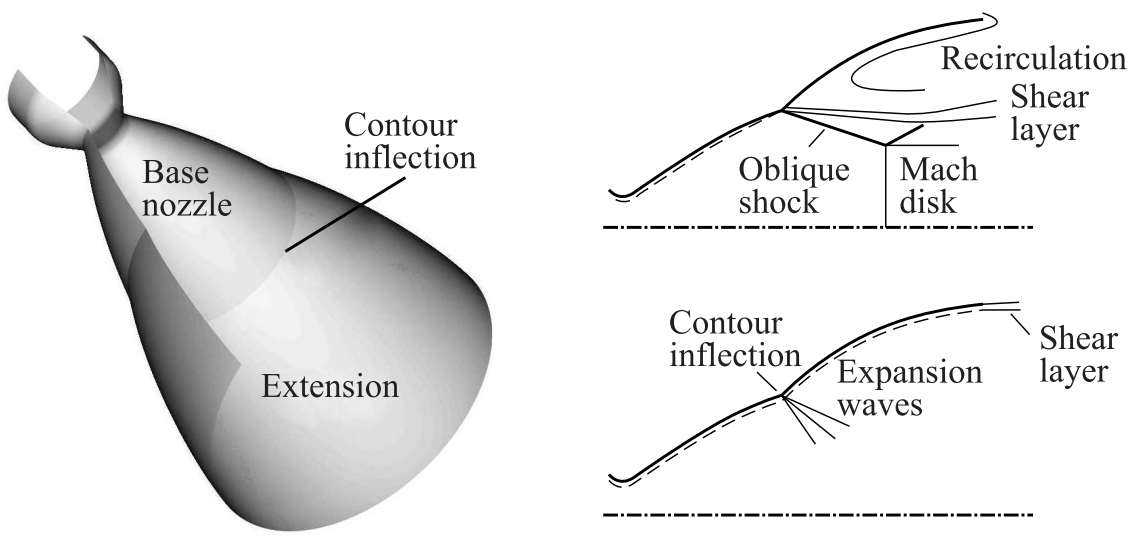

Figure 1 The dual bell nozzle and its two operating modes: sea level (top) and altitude mode (bottom)

corresponding to a given nozzle pressure ratio (NPR), the transition to altitude mode takes place: the flow suddenly attaches to the extension wall down to the nozzle exit plane. The nozzle extension flows full and the whole area ratio is used, leading to a higher vacuum performance.

The concept of a contour inflection to control the flow separation was first proposed in 1949 by Foster and Cowles [2]. Many experimental and numerical studies have been conducted since then to prove the altitude adaptation qualities of the nozzle concept. In 1994, Horn and Fisher [3] investigated the influence of the extension contour geometry on the flow behavior in the first experimental study. In Europe, the FESTIP research group realized a feasibility and performance analysis $[1,4]$. Various parametrical studies have also been realized to understand the flow phenomena and optimize the contour design $[5,6]$.

Cold flow subscale dual bell nozzle models have been tested intensively at the DLR [7]. Axisymmetrical nozzles were tested both under cold and hot flow conditions. Schlieren optics were used to investigate the flow evolution during the transition. For the present study, a 2D (planar) nozzle model has been chosen to permit the flow observation during the transition directly in the region of the contour inflection. The tests were conducted at the hot gas M11 test complex at DLR Lampoldshausen. In addition, a cold flow test campaign was conducted at the cold flow test facility P6.2, for the transient investigation.

Representative test cases were then chosen and recalculated with a computational fluid dynamics (CFD) tool. The comparison of the calculated results with the experimental data permits to validate the numerical method. Both experimental and numerical works were realized in the framework of the SFB-TR40 cooperation of the German Research Foundation. 


\section{EXPERIMENTAL SETUP}

The experimental study was divided into two campaigns: a cold flow investigation at the P6.2 test facility and a hot flow study at the M11 test complex. The cold flow installation offers a great flexibility in the measurement methods (wall pressure meausrements and schlieren optics) and transient test conditions, whereas the hot flow installation yield operation in conditions closer to reality.

\subsection{Cold Flow Test Facility P6.2}

The P6.2 test facility provides dry gaseous nitrogen obtained from $20 \mathrm{MPa}$ high pressure vessels. The flow gets through a line system with an automatic valve, a filter, a pressure reducer and a mass flow meter. It reaches the settling chamber including a combination of grids and honey combs to reduce the turbulence intensity. The test specimen is mounted on the horizontal rig, downstream of the settling chamber and a section contraction, delivering a parallel flow.

The test facility supplies the nozzle with a feeding pressure up to $6 \mathrm{MPa}$ and a mass flow up to $4.2 \mathrm{~kg} / \mathrm{s}$, under ambient temperature. The tests were conducted under ambient pressure conditions with up- and downramping of the feeding gas pressure $( \pm 0.1 \mathrm{MPa} / \mathrm{s})[8]$.

\subsection{Test Complex M11}

The test complex M11 is divided into four test benches designed for the investigation of ramjets, scramjets and small supersonic nozzles. The test position M11.1 features hydrogen-oxygen burners heating pressurized air. The four-burner configuration in use for this test campaign yields total pressures up to $3 \mathrm{MPa}$ and temperatures up to $1500 \mathrm{~K}$. Flow temperature and pressure can be adjusted by
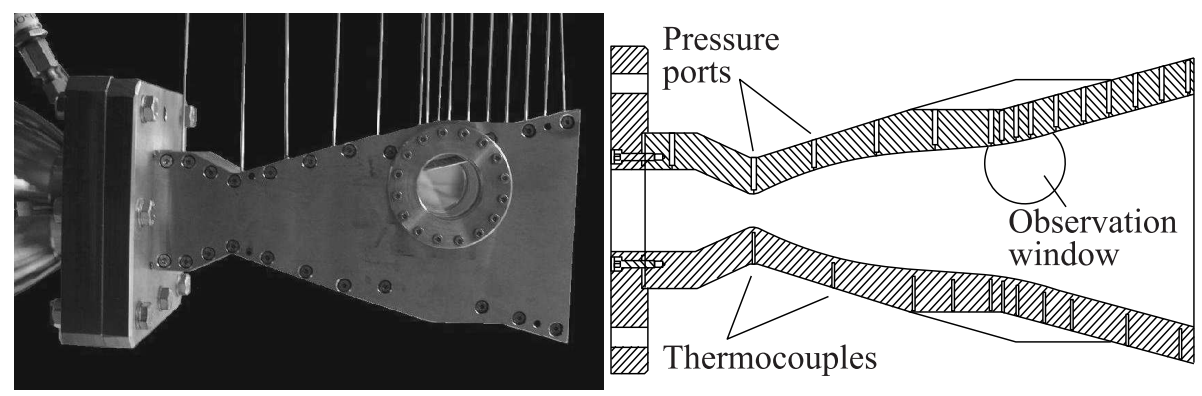

Figure 2 Planar dual bell nozzle model 


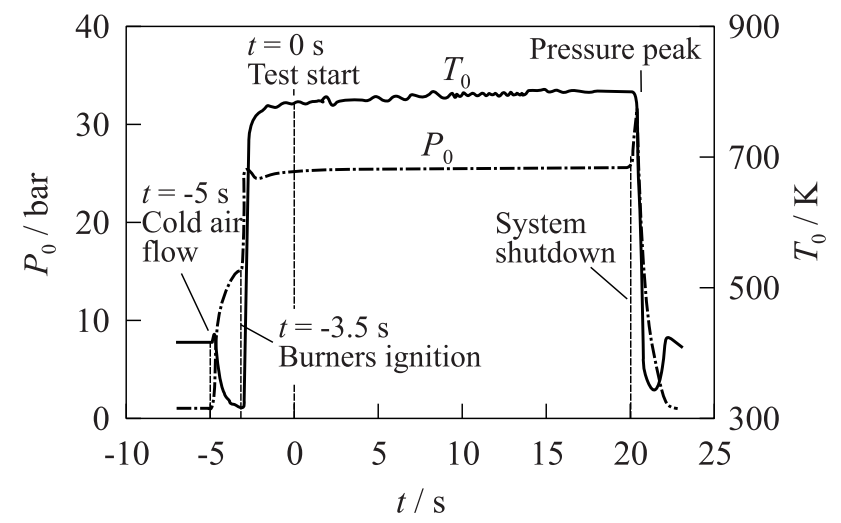

Figure 3 Typical total pressure and temperature variations during a hot flow test at M11

varying the hydrogen, oxygen and air mass flow rates. The hot flow was composed of air with about $5 \%$ water vapor for the test conditions presented in this study. The nozzle model was mounted on a horizontal rig (Fig. 2). The tests were conducted under ambient conditions in pressure and temperature. Figure 3 depicts a typical pressure and temperature evolution during a steady state test at test bench M11.1.

\subsection{Nozzle Model}

The test specimen was designed for the hot flow investigation at the M11 facility. The model was a planar dual bell nozzle. The base nozzle was designed as a full-length ideal nozzle, to limit the three-dimensional (3D) effects due to the side walls. The design Mach number was $\mathrm{M}_{D}=2.8$. The base nozzle flows

Table 1 Geometry of the dual bell nozzle model

\begin{tabular}{lc}
\hline \multicolumn{1}{c}{ Parameter } & Value \\
\hline Throat radius, $R_{\mathrm{th}}, \mathrm{mm}$ & 9 \\
Base length, $L_{b} / R_{\mathrm{th}}$ & 15.1 \\
Extension length, $L_{e} / R_{\mathrm{th}}$ & 11.9 \\
Area ratio, $\epsilon_{b}$ & 3.9 \\
Area ratio, $\epsilon_{e}$ & 7.1 \\
Inflection angle, $\alpha$ & $15^{\circ}$ \\
Depth of the model, $d, \mathrm{~mm}$ & 45 \\
\hline
\end{tabular}




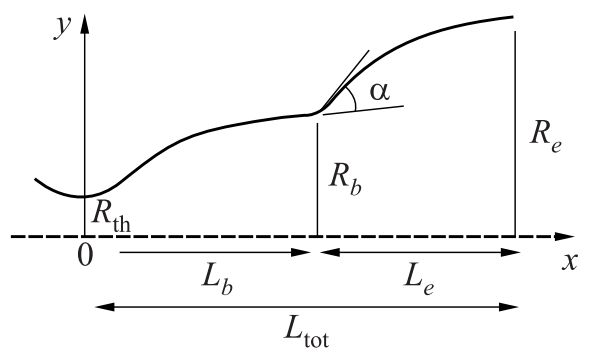

Figure 4 Geometrical parameters of a dual bell nozzle

full for NPR 13 and is adapted for NPR 30. The extension was defined as an isobar leaving the last point of the base nozzle (constant pressure extension). The design in-house program is based on the method of the characteristics. The theoretical transition nozzle pressure ratio is $\mathrm{NPR}_{\mathrm{tr}}=P_{0} / P_{a}=25.8$. The nozzle extension is adapted for NPR 90. The geometrical parameters are summarized in Table 1 and illustrated in Fig. 4.

The nozzle was constituted of two walls corresponding to the nozzle contour and two exchangeable parallel side plates. The nozzle depth was $45 \mathrm{~mm}$ and constant from the convergent nozzle part down to the exit plane. The wall thickness for the side plates and the nozzle contours was $10 \mathrm{~mm}$.

\subsection{Instrumentation}

Wall pressure measurements were performed for the nozzle model with piezoresitive "Kulite Semiconductor Inc." transducers (model XT-154-190M). The pressure ports were placed along the nozzle wall, in the center line of the upper nozzle contour. Small pipes welded in the wall connected the sensors to the 0.5-millimeter diameter orifices via small Teflon tubes (see Fig. 2). In addition, the pressures were measured in the side plates inside a port matrix placed in the observation window, permitting the comparison with the schlieren measurements. The transducers have a range of $0.1 \mathrm{MPa}$ and an accuracy of $0.5 \%$ in this operating range. The signals were recorded with a rate of $1 \mathrm{kHz}$ and were low-pass filtered at $160 \mathrm{~Hz}$ for the cold flow transient tests.

A window of 45-millimeter diameter was located in the region of the contour inflection of the side plates. The flow evolution in this area has been observed using schlieren optics. Color schlieren films were recorded for up- and downramping of the NPR at the cold flow test bench. The density gradient variations of the flow in the inflection region visualize the separation shock system. 


\section{NUMERICAL METHOD}

The cold flow tests provide the possibility of a better instrumentation (transient pressure measurements and schlieren optics). For this reason, cold flow test cases were selected for the numerical simulation using the commercial 3D NavierStokes solver Ansys CFX.

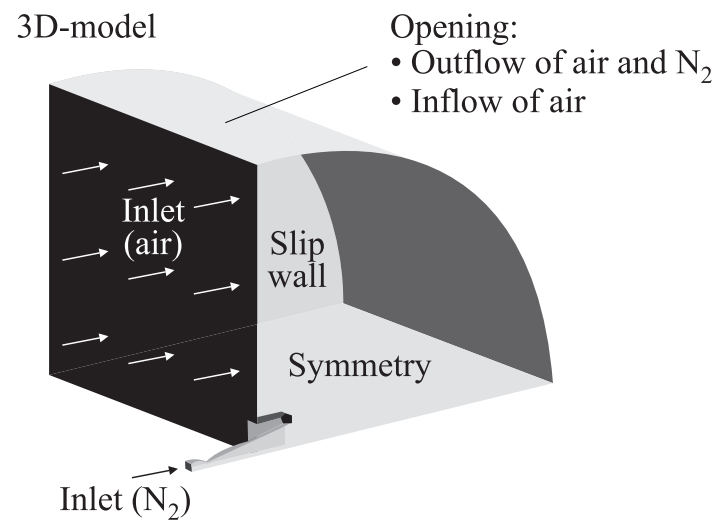

Figure 5 Boundary conditions for numerical simulation

The numerical model for the simulation of the planar dual bell nozzle and the boundary conditions are depicted in Fig. 5. The fluid is nitrogen as a working medium for the nozzle and air for the ambience. Due to symmetry reasons, only one fourth of the nozzle is simulated and symmetry boundary conditions are used at the corresponding planes. At the nozzle inlet, a total pressure in the range of 1.3 to $2.7 \mathrm{MPa}$ and a total temperature of $293 \mathrm{~K}$ were prescribed. The nozzle walls were set as adiabatic and assumed to be hydraulically smooth. Around the nozzle, a control volume was constructed in order to take into account the interaction with the ambience. The grid resolving the depicted computational domain contained about 4 million nodes. At the control volume boundary in the direction of the nozzle inlet, an inflow boundary condition with ambient total pressure and temperature $\left(P_{0}\right.$ $=0.1 \mathrm{MPa}$ and $T_{0}=300 \mathrm{~K}$ ) is used. In the direction of the nozzle outlet, an opening boundary condition with prescribed total pressure and temperature is applied, which allows the outflow of air and nitrogen and the inflow of air. As a turbulence model of the steady-state Reynolds-Averaged NavierStokes (RANS) simulations, the Menter Shear Stress Transport (SST) model was used. 


\section{RESULTS AND DISCUSSION}

\subsection{Flow Evolution in the Nozzle}

The wall pressure has been recorded along the nozzle contour for the various test configurations obtained at the test rig M11. Figure 6 depicts the wall pressure distribution for four values of NPR measured for the same total temperature $T_{0}=700 \mathrm{~K}$.

For low NPR values (NPR $=20$ and 24.2), the nozzle model is operating in sea level mode. The flow is attached in the base nozzle and separates at the contour inflection. When increasing the NPR value (24.7), the separation point starts moving into the extension nozzle. This phenomenon has been described as sneak transition [6].

However, when further increasing the NPR value, the separation point displacement continues progressively down to the nozzle exit. The expected abrupt transition to high altitude mode does not take place. This phenomenon indicates that the extension wall contour features a small negative pressure gradient. It seems that the design code developed for axisymmetric nozzles is not adapted for planar nozzles which present strong $3 \mathrm{D}$ effects.

The negative wall pressure gradient up to $x / r_{\text {th }}=20$ followed by a positive wall pressure gradient is confirmed by a CFD simulation at altitude mode (Fig. 7). Therefore, a distinct sneak transition takes place prior to the transition to the nozzle full-flowing.

Test cases of different separation types were simulated with the commercial CFD solver for the cold flow conditions. At a low nozzle pressure ratio (NPR

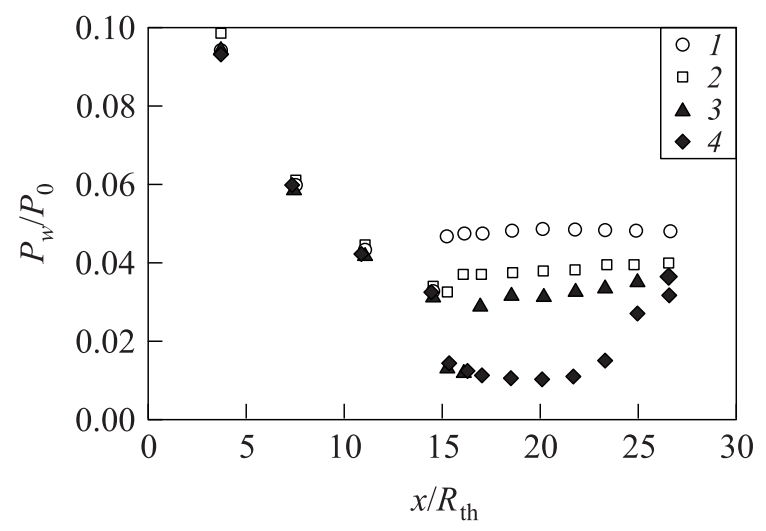

Figure 6 Wall pressure distribution for various NPR values $\left(T_{0}=700 \mathrm{~K}\right): 1-20$; $2-24.2 ; 3-24.7$; and $4-29.9$ 


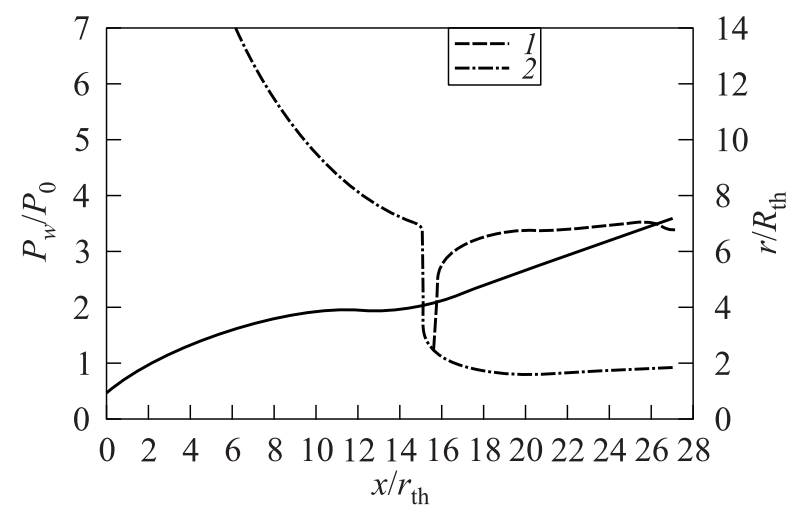

Figure 7 Wall pressure evolution in sea-level (1) and vacuum (2) modes for cold flow simulation

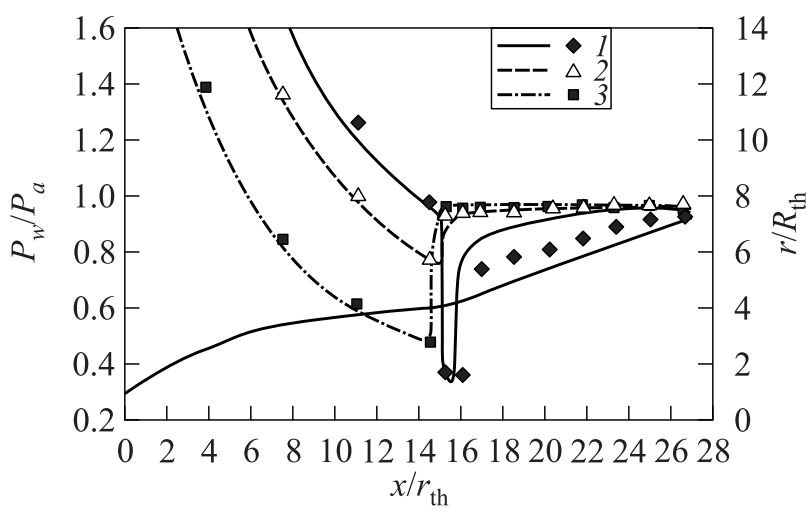

Figure 8 Comparison of wall pressure evolution in test (signs) and simulation (curves) for cold flow conditions: $1-\mathrm{NPR}=27.2 ; 2-21.9$; and $3-\mathrm{NPR}=13.5$

$=13.5$ ), flow separation takes place in the base nozzle, as depicted in Fig. 8. As the NPR is increased, the separation moves to the contour inflection (NPR $=21.9)$ and is finally located in the region of the sneak transition (NPR $=27.2)$. The agreement between the test and the simulation is good for the two lower nozzle pressure ratios. For the highest nozzle pressure ratio, the agreement is not as good as for the other ones. The flow separation occurs slightly further upstream than in the test, which is already known from simulations of conventional nozzles. Additionally, the plateau pressure downstream of the flow separation is higher in the simulation than in the test. 


\subsection{Schlieren Observation}

The schlieren pictures were recorded with a rate of 50 frames per second. Figure 9 shows a series of four schlieren pictures taken during a cold flow transient test. The total pressure was progressively increased to observe the flow evolution during transition.

Figures $9 a$ and $9 b$ were taken for NPR $=11.73$ and 16.38. The separation shock system is located directly at the inflection: the nozzle flows in sea-level mode. Figure $9 c$ corresponds to a NPR value of 25.02. The separation point has started to move into the extension: the sneak transition is taking place. In

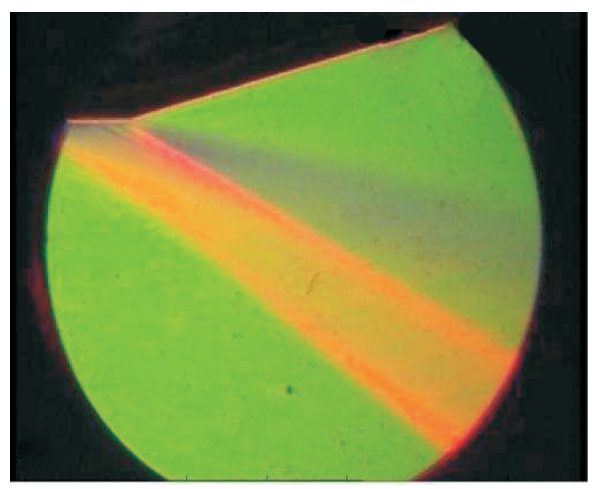

(a)

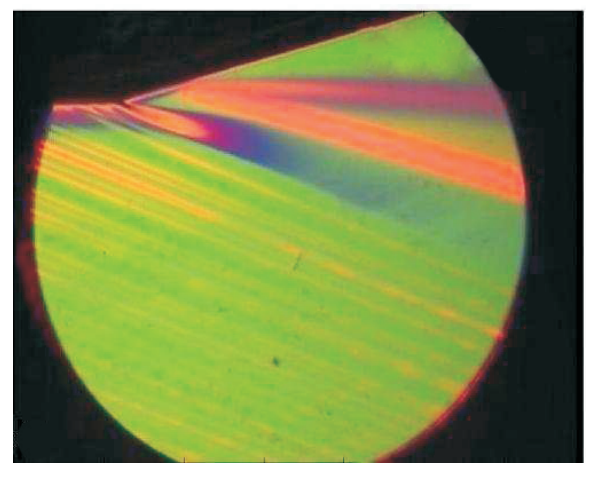

(c)

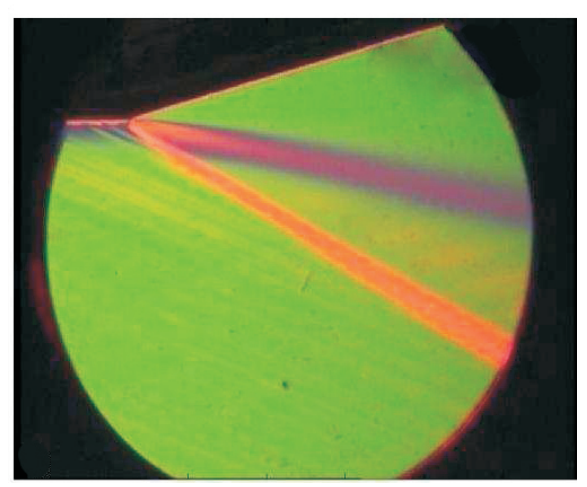

(b)

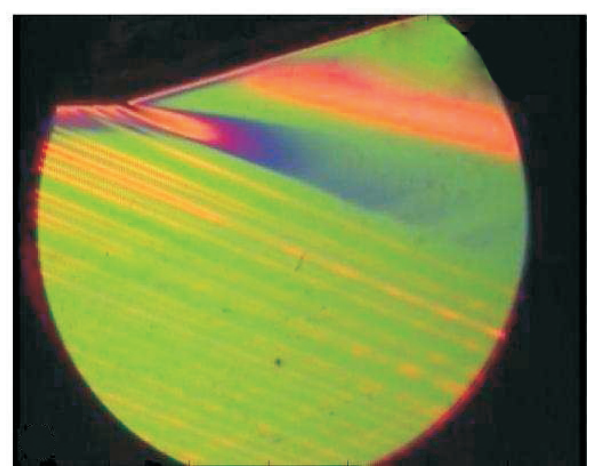

$(d)$

Figure 9 Observation of the flow in the extension region with color schlieren: (a) NPR $=11.73 ;(b) 16.38 ;(c) 25.02$; and $(d) \mathrm{NPR}=27.37$. Upper row refers to sea-level mode and lower to sneak transition. 


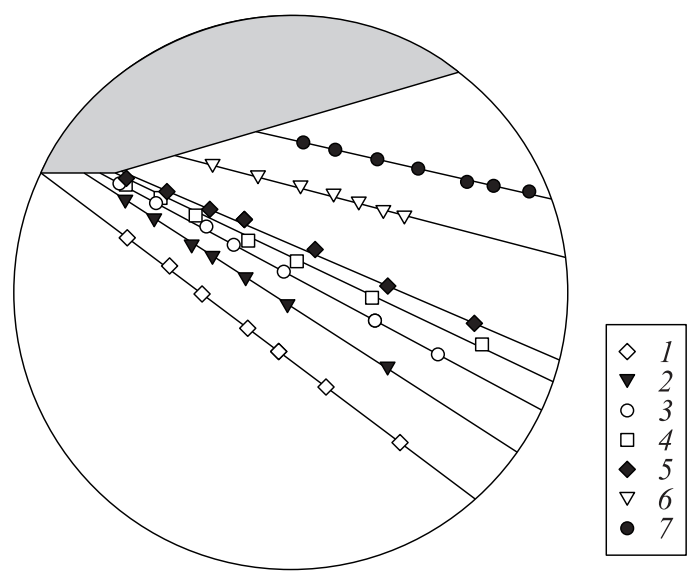

Figure 10 Evolution of the separation angle during NPR up- and downramping: $1-\mathrm{NPR}=11.9 ; 2-13.5 ; 3-14.5 ; 4-21.9 ; 5-23.1 ; 6-24.8 ;$ and $7-$ $\mathrm{NPR}=27.2$

Fig. $9 d(\mathrm{NPR}=27.37)$, the separation point has moved further downstream into the extension.

The slight negative wall pressure gradient in the extension leads to a slower transition from sea level to altitude mode. This effect yields the possibility to observe in detail the evolution of the separation shock system. The position and the shape of the separation shock have been measured from the schlieren pictures using an in-house tracing program. Figure 10 illustrates the position of the separation shock in the observation window for various NPR values, from sea level mode to almost altitude mode.

The angle between the separation shock and the extension wall has been recorded while varying the NPR value. As the separation shock shows slight variations due to unsteadiness of the separation point, the angle value has been taken as an average of various pictures corresponding to the given conditions. At a low NPR, the flow separates in the base nozzle. The angle decreases progressively while the jet opens. In sea level mode, the separation point is fixed at the contour inflection and the jet continues widening. Once the separation point starts moving into the extension, the angle remains almost constant. A similar effect can be seen when lowering the NPR, like during the retransition. The evolution of the jet angle is linked to the shape of the recirculation area in the extension in sea level mode, and hence, the pressure downstream of the separation point.

The second angle is defined between the horizontal and the separation shock, as illustrated in Fig. 11. The values of the measured angles were then compared to the CFD simulations, where the angles were determined by contour plots of 


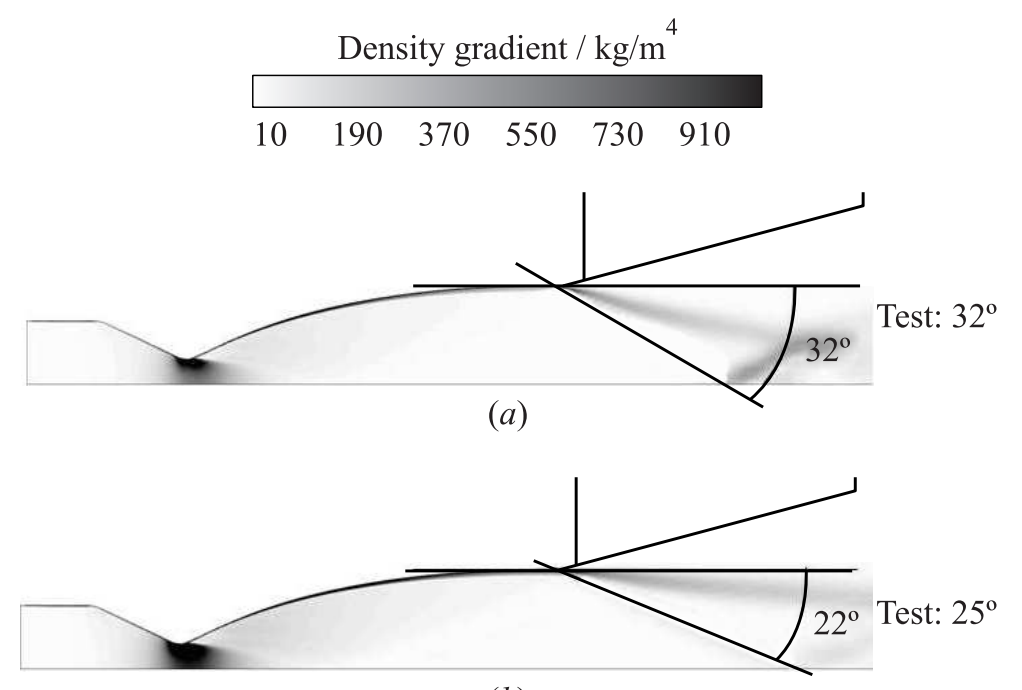

(b)

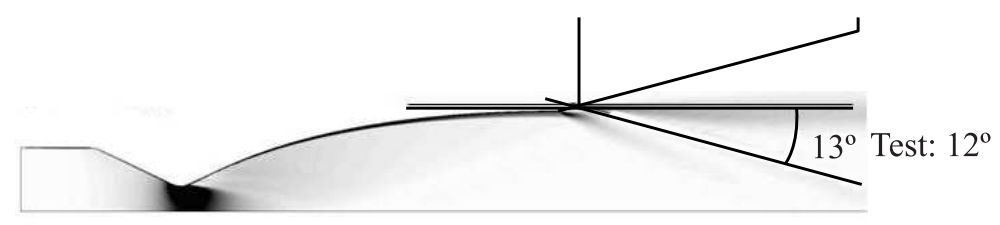

(c)

Figure 11 Evaluation of shock angle with density gradient contour plots: (a) NPR $=13.5 ;$ (b) 21.9; and $(c) \mathrm{NPR}=27.2$

the density gradient. Although the evaluation of the angle is not very precise, it, nevertheless, gives a hint for the modeling capability of the shock. In all investigated test cases, the angles of CFD and the test were close to each other (angle difference $\leq 3^{\circ}$ ), even in the case where slight differences in the wall pressure were present (e.g., for NPR 27.2).

\subsection{Three-Dimensional Effects}

The test facility M11.1 limits the maximal depth for a $2 \mathrm{D}$ nozzle to $d=45 \mathrm{~mm}$. The depth over width ratio, $d / R_{\mathrm{th}}=2.5$, is rather low at the nozzle throat and becomes very small at the nozzle exit $d / R_{\mathrm{th}}=0.35$. This leads to a $3 \mathrm{D}$ behavior of the flow in the nozzle. One effect can be seen in Fig. $12 a$, a schlieren picture of the flow for a low NPR value. 


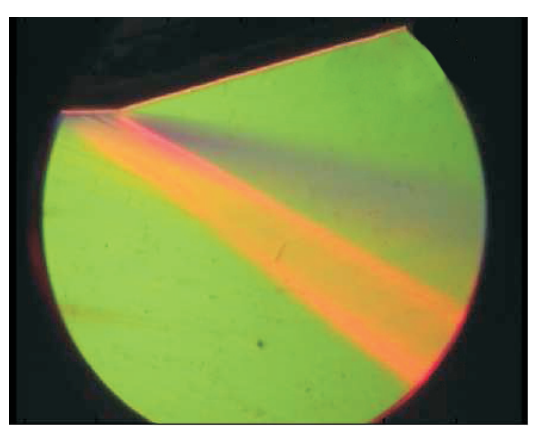

(a)

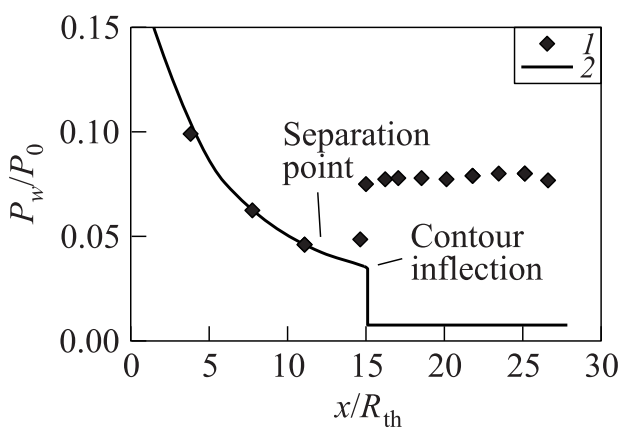

(b)

Figure 12 Three-dimensional effect in sea level mode: comparison of schlieren picture $(a)$ and wall pressure measurement for NPR $=11.7(b): 1$ - experimental data; and 2 - vacuum profile.

The separation shock appears to be very thick, from a position in the base nozzle down to the inflection. The graphic in Fig. $12 b$ is the wall pressure distribution corresponding to the schlieren picture. The flow separates in the center line of the nozzle contour at the most upstream position. On the side walls, the flow is already attached at the inflection, forming a "U"-shaped flow pattern.

In Fig. $13 a$, where the separation line $\left(\tau_{\text {wall }}=0\right)$ is depicted, a similar behavior can be observed in the numerical simulation at low NPR: the flow separates at the centerline upstream of the contour inflection, while it is already attached to
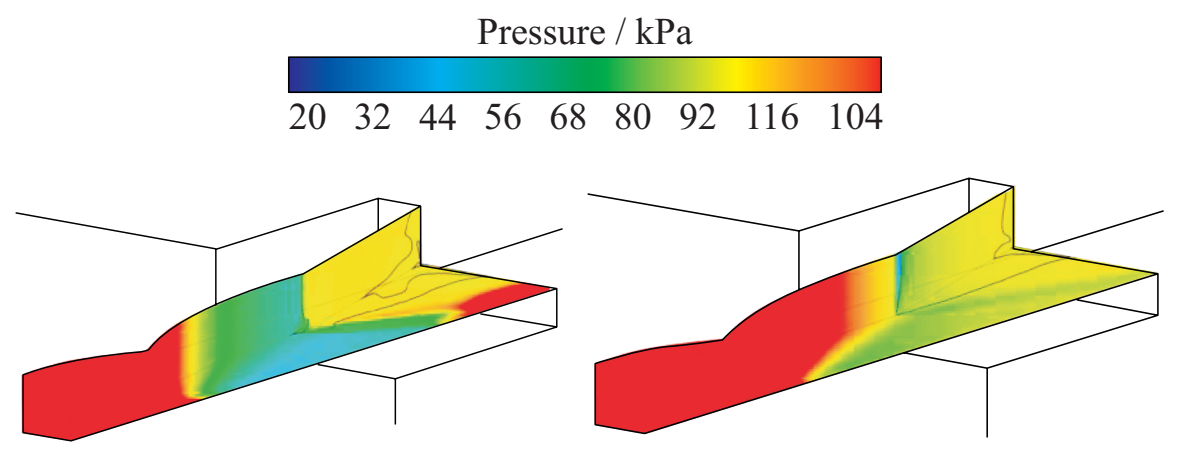

Figure 13 Three-dimensional shape of separation line $\left(\tau_{\text {wall }}=0\right):(a)$ NPR $=13.5$; and $(b) \mathrm{NPR}=27.2$. 
the contour inflection in the region close to the side plate. However, for higher NPR, the situation changes (Fig. 13b). In the corner between the side plate and the contoured wall, the flow separation takes place directly at the contour inflection, while in the nozzle symmetry plane the flow is already in the sneak transition mode.

\section{CONCLUDING REMARKS}

A planar dual bell nozzle model has been tested under various test conditions in cold and hot flow. The observation of the shock system in the region of the contour inflection gives information on the position and the shape of the separation front. In sea-level mode, the experimental and numerical results are in good agreement. For higher NPR values, the calculated separation position is located further upstream than measured in the experiments. Despite these small differences with the experiments, the numerical method yields a good simulation from the flow behavior and helps to improve the understanding of the physics of the flow. In sea-level mode, the simulation provides additional information on the shape and position of the separation line, which cannot be directly measured experimentally due to the $3 \mathrm{D}$ effects of the side walls.

\section{ACKNOWLEDGMENTS}

Financial support has been provided by the German Research Foundation (DFG - Deutsche Forschungsgemeinschaft) in the framework of the Sonderforschungsbereich Transregio 40.

\section{REFERENCES}

1. Immich, H., and M. Caporicci. 1996. FESTIP technology developments in liquid rocket propulsion for reusable launch vehicles. AIAA Paper No. 1996-3113.

2. Foster, C., and F. Cowles. 1949. Experimental study of gas-flow separation in overexpanded exhaust nozzles for rocket motors. Progress Report No.4-103. Jet Propulsion Laboratory.

3. Horn, M., and S. Fisher. 1994. Dual-bell altitude compensating nozzles. Rocketdyne Division. NASA-CR-194719.

4. Hagemann, G., M. Frey, and D. Manski. 1997. A critical assessment of dual bell nozzles. AIAA Paper No. 1997-3299.

5. Martelli, E.. F. Nasuti, and M. Onofri. 2007. Numerical parametric analysis of dual-bell nozzle flows. AIAA J. 45:640-50. 
6. Génin, C. 2010. Experimental study of flow behaviour and thermal loads in dual bell nozzles. Ph.D. Thesis. Université de Valenciennes, France.

7. Nürnberger-Génin, C., R. Stark, H. Ciezki, and O. Haidn. 2009. Experimental study of transition behaviour in high adaptive dual bell nozzles. Annual Report SFB/TRR 40.

8. Frey, M., R. Stark, H. Ciezki, F. Quessard, and W. Kwan. 2000. Subscale nozzle testing at the P6.2 test stand. AIAA Paper No. 2000-3777. 